INTERNATIONAL DESIGN CONFERENCE - DESIGN 2018

https://doi.org/10.21278/idc.2018.0511

\title{
PREDICTION OF THE RESIDUAL LIFE OF A COMPONENT UNDER INTENSIVE RANDOM DYNAMIC LOADING WITHIN THE SCOPE OF TECHNICAL INHERITANCE
}

\author{
I. Mozgova, I. Yanchevskyi and R. Lachmayer
}

\begin{abstract}
An important part of the Technical Inheritance approach are suited methods and algorithms for analyzing the data of manufacturing and usage of components, as well as methods for assessing the current state of the component during operation and predicting its residual life. In this paper we are going to describe the concept of TI as a process model and the main challenge of how to collect and analyse data, paying special attention to one aspect of the approach - forecasting the residual resource of a component under conditions of intense random loading.
\end{abstract}

Keywords: design methods, data driven design, design knowledge

\section{Introduction}

The concept of Technical Inheritance (TI) is devoted to the development of an approach of collecting and analysing data at different phases of the product life cycle with the aim of developing, producing and operating new generations of a product based on the life cycle data of previous generations which are more adapted to new requirements of the actual environment.

Special attention should be paid to the process of designing new product or component generations by using collected data and structured information obtained as a result of their analysis. It has to be taken into account, that many components in operation are subjected to conditions of a complex dynamic or cyclic loading. Frequently, the causes of premature failure of such elements are short-term peak loads or accumulated fatigue damage. Even if at the design stage of a component various measures to reduce the effect of peak loads on the reliability of the component can be taken, a warning regarding the occurring fatigue failure at this stage is a fairly complex task.

In the following, we describe an integrated approach to develop, produce and operate products or components, called TI, which includes CAD modeling of the component, optimization of the model using methods of data analysis and the finite element method and taking into account usage data about loads of previous generations of the component as well as analysed data obtained during monitoring the manufacturing and usage of the component. The process of fatigue accumulation and material degradation can be tracked during the operation of a component by monitoring and controlling the planned diagnostic parameters. Within the framework of the presented methodology, based on the data collected at the operational stage, the residual life of the component under intensive random loading can be predicted and the information relevant for the development of next generations of components can be recognized. 


\section{Paradigm of Technical Inheritance}

One central point of the fourth industrial revolution are smart systems, products or components that are configured or adapted to new tasks, that serve and analyse themselves and can change their processing depending on the environment's requirements. Mechanisms and methods for collecting and analysing data in such components must be provided already at the stage of the development.

$\mathrm{TI}$ is defined as a transfer of assembled and verified information from manufacturing, usage and application to the next component generation (Lachmayer et al., 2014a). The purpose of TI is the development of new generations of a component adapted to new or changed requirements on the basis of information from the development, manufacturing and exploitation of the existing generations of the product or component. Hence, we can speak of the evolution of technical products or components. By means of the processes of TI, information should be generated in a product-specific manner, which in this way creates a great advantage for the development of new generations of a component. Evolutionary adaptation of the technical system includes an inverse information relationship, providing a suitable response of complex self-controlled systems to changing environmental conditions. In the general context of TI, adaptation is the development of a new generation of components, adapted to new requirements of the environment and the user. The information is stored in appropriate databases. Each new generation of a component, passing in its life cycle through the phases of development, manufacturing and usage, generates its own information (Figure 1).

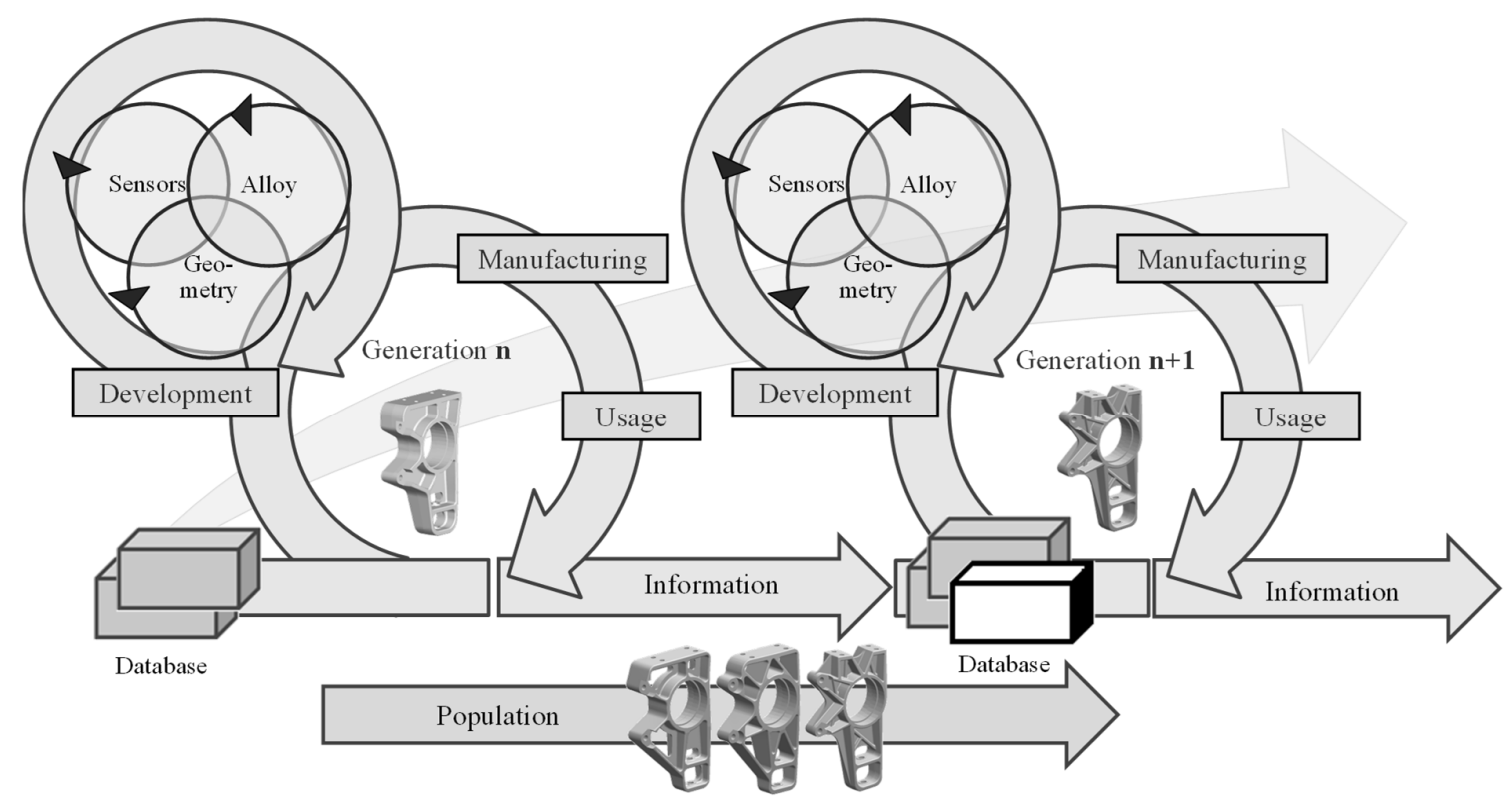

Figure 1. Evolutionary adaptation of a component (Mozgova et al., 2017)

Each population consists of several generations of a product. A generation is the group of individuals with the same level of development. The individual is the smallest considered technical system, product, component, process or model in a population (Lachmayer et al., 2015; Demminger et al., 2016).

Since methods for collecting data of the manufacturing and usage of a component are considered within the context of TI, the development process itself is iterative and includes the phases of developing and optimizing the geometry of the component as well as selecting appropriate materials and types and locations of sensors. The sensors can be inherent, for example, when using components made of materials with sensory properties, or can be external (Barton, et al. 2016; Gottwald, 2016).

Each phase of development is iterative. Thus, by developing and optimizing the geometry of a component, material properties are taken into account. The choice of the sensor type, in turn, depends on the material and the geometry of the component. The position of the sensors, for example on the component surface, should be planned not only to take into account locations of critical or characteristic 
loadings, but also the sensor availability regarding data reading and preventive maintenance, repair or replacement. This in turn can require changes in the component's geometry.

An important challenge in linking and reusing information lies in the definition of a flexible data format, since additional information forms have to be integrated into the existing format during the lifecycle as well as in subsequent component generations. For a structured information exchange, an XML-based data exchange format can be used, which features a hierarchical structure and includes different levels: a component level (component in complete condition), a functional assembly level, a component level and a design element level. The application of a developed XML-based format for data exchange is described more in detail in (Denkena et al., 2016).

\subsection{Collecting data and monitoring the current state of the component}

The general scheme of the approach to develop a new generation of a component based on data collected during the exploitation of previous generations is depicted in Figure 2. The approach involves taking into account manufacturing restrictions in the context of product and component development (Gembarski et al., 2016), analysing data of the component usage (Lachmayer et al., 2014b), monitoring and controlling the current state of the component as well as analysing the results of usage (Schubert et al., 2013). By monitoring, carried out primarily for the main components, the current technical conditions can be evaluated and the residual lifetime of the element can be predicted.

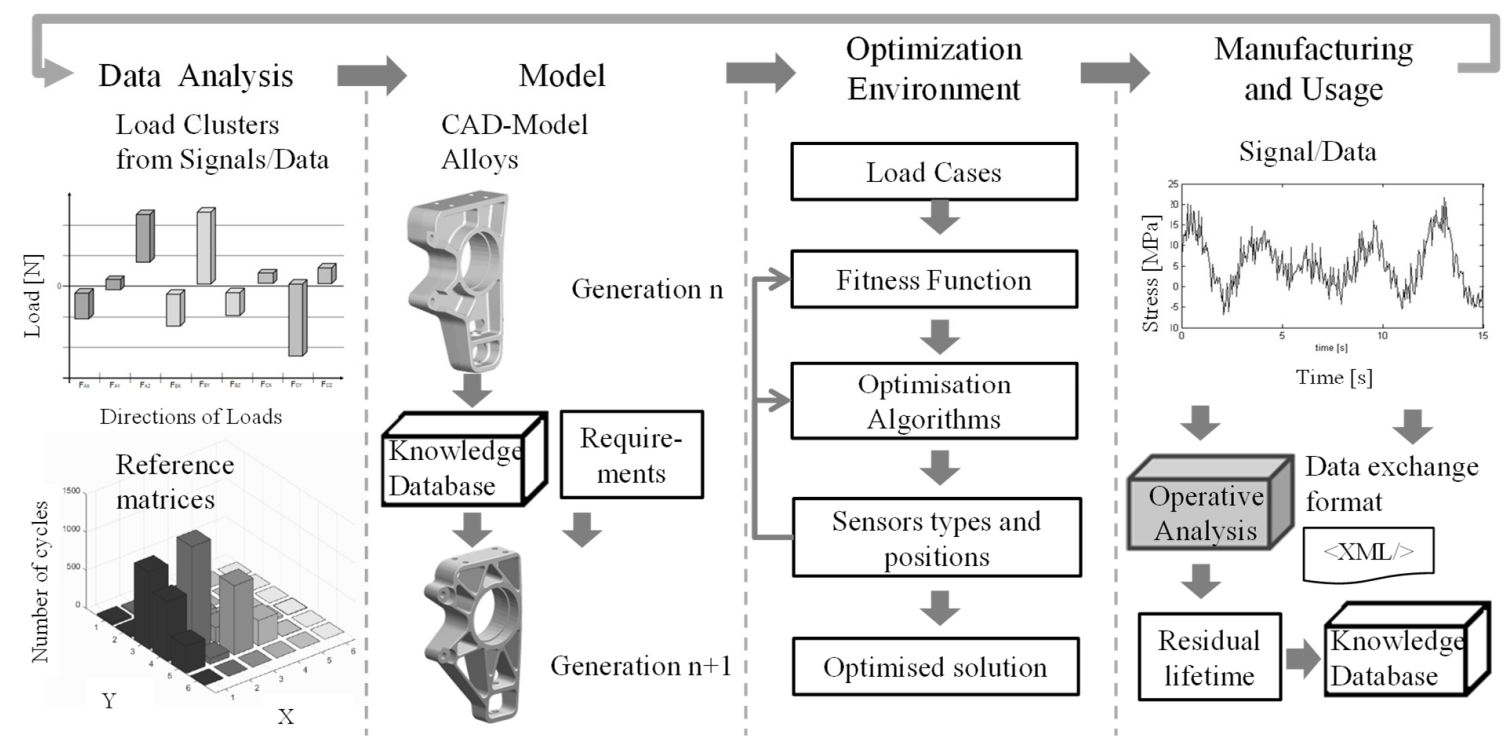

Figure 2. Algorithmized information feedback from the product life cycle

The effectiveness of this approach was illustrated at the example of an information based data feedback and the development of a new generation of a wheel carrier (Lachmayer et al., 2013). Currently, aspects of storage of relevant information by using this approach in the context of the development and operation of components, which have the ability to collect data, for example, using additional intelligent units, are being studied in (Scheidel et al., 2017).

The approach involves testing the component geometry during the usage phase or on the test bench, analysing collected data and developing a new generation of the component, specifying the type and location of the loads, the types, directions and places of force application and the kind and sizes of the moments based on the analyzed data. During the development process of a new generation, a selection of suited materials and the type and location of the sensors as well as choosing or correcting the data modeling and analysis algorithms is required. 


\section{Operative analysis of the state of the component and a method for prediction of the residual lifetime}

Most of the components of modern driving systems are operated under complex dynamic loading conditions. Even if it is possible to provide various methods and technologies to reduce the effect of peak loads on the reliability of elements at the design stage, it is a fairly complex task to predict and prevent fatigue failure by suited measures at this stage.

The process of material degradation, which is the subject of study of many scientists (Zhou et al., 2011; Bian et al., 2015) can be tracked during the operation phase of structural elements through the control of relevant diagnostic parameters (Goto and Tsukamoto, 2012; Bian et al., 2015).

By means of such a control, which is carried out primarily for indispensable structural components, it is possible not only to estimate the current technical state, but also to predict the residual lifetime of the component by extrapolating the analytical dependencies approximating the history of change of the diagnosed parameters under the assumption that the maximum permissible values are known.

This article is devoted to this problem. The solution of this problem is based on previously published results (Lachmayer et al., 2018), which describe the method of identifying loads on the component according to suited sensory data.

\subsection{Formulation of the problem}

Calculation of the measure of accumulated fatigue micro damages $D$ is carried out for a part whose geometry, mechanical material characteristics, attachment variants and the position of the system of $R$ applied external forces $F_{r}(t)$, are given, where $t$ denotes the time.

The laws of time variation of these forces are not known, but it is believed that they can be recovered from the readings of $S$ sensors recording the deformation of the component at $S$ positions according to the functions $E_{S}(T), s=\overline{1, S}$. The task is to develop a methodology for calculating the amount of accumulated fatigue damage $D$ of a component during its operation such that the functions $E_{S}(t)$ are known, and all dynamic processes are linear. It should be noted that the residual life of the component can be indirectly estimated by the criterion of its fatigue strength that is the current value of the parameter $D$, where its limiting value equals 1 .

\subsection{Endurance calculation}

The classical methodology for calculating multicycle fatigue of a component subject to random, irregular loading is based on the assumption that the entire loading history is a collection of identical blocks within which the functions $F_{r}(t)$ are known. In engineering practice, the most widespread is the linear model of summation of fatigue damages of Palmgren-Miner (Collins, 1993).

The measure of damage in the material of a component in the calculation area for a single loading block is designated as a dimensionless quantity $\Delta D$. The calculation of $\Delta D$ for a particular region is carried out by means of the history of the change of the equivalent stress $\sigma_{e}(t)$ in the time block with the duration $\Delta T$, which is due to the action of the external loads $F_{r}(t)$. The function $\sigma_{e}(t)$ can be calculated using methods of mathematical modeling.

Since most of the experimental data, for which further calculations were made, was obtained for the symmetric regular changes of equivalent stress during the time, the first stage involves a transfer from the original history of $\sigma_{e}(t)$ to an uniaxial equivalent stress $\hat{\sigma}$ with the same damaging action. For this, using the rainflow counting method (Köhler et al., 2012), the history of equivalent stress $\sigma_{e}(t)$ is schematized by replacing the original random function to an ordered step-function with fixed parameters. Thus, we obtain a matrix containing detailed information about the allocated stress blocks. This matrix depicts the original history $\sigma_{e}(t)$ as a set of asymmetric modes of equivalent stress variation. 
In the second stage, the conversion to the equivalent (in the sense of the inserted fatigue damage) symmetric mode is performed. As a result, we get a new search history of the stress change $\hat{\sigma}$, which has the form of a stepped histogram, within each step of which the equivalent stress is regular.

At the final stage, a direct calculation of fatigue damage $\Delta D$ is possible and the approximate number of blocks before appearance of fatigue failure can be determined by the ratio $\lambda=1 / \Delta D$.

With a random dynamic load whose time variation can't be represented as a collection of periodically repeating identical blocks, the calculation of fatigue strength is often carried out by means of the probabilistic laws of distribution of the loading maxima.

An alternative approach assumes a direct identification of the values of these loads according to the data from corresponding sensors. In accordance with the approach described by Lachmayer et al. (Lachmayer et al., 2018), the identification of a system from $R$ unknown forces can be realized in real time, assuming that dynamic processes can be considered quasi-static, according to the data from $S$ sensors whose values are an indirect demonstration of the sought forces. In this case, the transformation formula for each step in time $m=1 . . M, M=\Delta T_{j} / \Delta t$, can be written as follows in the matrix form:

$$
\mathbf{F}_{m}=\left(\mathbf{A}^{\mathrm{T}} \mathbf{A}+\alpha \mathbf{C}\right)^{-1} \mathbf{A}^{\mathrm{T}} \cdot \mathbf{E}_{m}
$$

Here, the index $\mathrm{T}$ denotes the transpose operation, where $\mathbf{E}_{m}$ and $\mathbf{F}_{m}$ are column matrices with sizes $S$ and $R$ containing the sensor data; $\mathbf{A}$ is the $(S \times R)$-matrix of the sensitivity coefficients $a_{S, r}$, which reflect the response of the $S$-ith sensor to a single $r$ action, $a_{s, r}=E_{S}$ if $F_{r}=1$ and $F_{j \neq r}=0 ; s=\overline{1, S}$, $r, j=\overline{1, R}, \alpha \geq 0$ is the parameter of regularization, $\mathbf{C}$ is the unit matrix $R \times R$.

The elements of the matrices $\mathbf{E}_{m}, \mathbf{F}_{m}$ and $\mathbf{A}$ are defined by the expressions:

$$
\mathbf{E}_{m}=\left[\begin{array}{c}
E_{1}(m \Delta t) \\
E_{2}(m \Delta t) \\
\vdots \\
E_{S}(m \Delta t)
\end{array}\right] ; \mathbf{F}_{m}=\left[\begin{array}{c}
F_{1}(m \Delta t) \\
F_{2}(m \Delta t) \\
\vdots \\
F_{R}(m \Delta t)
\end{array}\right] ; \quad \mathbf{A}=\left[\begin{array}{cccc}
a_{1,1} & a_{1,2} & \ldots & a_{1, R} \\
a_{2,1} & a_{2,2} & \ldots & a_{2, R} \\
\vdots & \vdots & \ddots & \vdots \\
a_{S, 1} & a_{S, 2} & \ldots & a_{S, R}
\end{array}\right]
$$

The procedure for calculating the fatigue strength for such a random, non-periodic external loading can be constructed on the fact that the usage of the component is divided into finite time intervals with the duration $\Delta T_{j}$, for which the history of the stress $\sigma_{e j}$ changes can be formed for the selected area of the component.

On the basis of the assumption that the fatigue damages are of a cumulative nature, the measure of the total fatigue damage $D$ in the investigated area of the component for the current time of operation of the component $T=\sum_{j} \Delta T_{j}$ will be the sum $\Delta D_{j}$ of the values calculated in the previous steps $D=\sum_{j} \Delta D_{j}$

As a result, the residual life of the component can be indirectly estimated by the minimum value of the difference $1-D$.

The calculation procedure of $\Delta D_{j}$ assumes the existence of information about the history $\sigma_{e j}(t)$ in the $j$-th time interval with the duration $\Delta T_{j}$. Obviously, the law of its change in this case can't be predicted in advance, and it is rather problematic to calculate $\Delta D_{j}$ for all positions of the component. Justified is the calculation only for the most loaded zones of the component, in which the probability of fatigue failure is maximal. Obviously, there can be several such zones. The positions of these zones can be established both from the experience of operating this component and by the finite element method. The implementation of this approach will be described in the next paragraph at the example of a wheel carrier of a racing car. The calculation of the stresses $\sigma_{e j}(m \Delta t)$ can be substantially simplified if we use the assumption that the reaction of the parameters of the stress-strain state to external influences is linear and the superposition principle is valid. 
If we relate the component under consideration to a Cartesian coordinate system $O x y z$, and denote the stress state at the investigated point under the action of the $r$-th unit force $\left(F_{r}=1 ; F_{j \neq r}=0\right)$ by the vector $\overline{\boldsymbol{\sigma}}^{(r)}=\left\{\overline{\boldsymbol{\sigma}}_{x x}^{(r)} \overline{\boldsymbol{\sigma}}_{y y}^{(r)} \overline{\boldsymbol{\sigma}}_{z z}^{(r)} \overline{\boldsymbol{\sigma}}_{x y}^{(r)} \overline{\boldsymbol{\sigma}}_{y z}^{(r)} \overline{\boldsymbol{\sigma}}_{x z}^{(r)}\right\}$, where $\overline{\boldsymbol{\sigma}}_{*}^{(r)}$ are components of the stress tensor, then the stress state at this point, determined by the stress tensor $\boldsymbol{\sigma}(t)=\left\{\sigma_{x x}(t) \sigma_{y y}(t) \sigma_{z z}(t) \sigma_{x y}(t) \sigma_{y z}(t) \sigma_{x z}(t)\right\}$ will be a linear combination of stress states $\overline{\boldsymbol{\sigma}}^{(r)}$ :

$$
\boldsymbol{\sigma}(t)=\sum_{r=1}^{R} F(t) \overline{\boldsymbol{\sigma}}^{(r)} .
$$

The equivalent stress can be calculated as

$$
\sigma_{e}=\operatorname{sign}\left(\sigma_{1}+\sigma_{3}\right) \sqrt{\left(\left(\sigma_{1}-\sigma_{2}\right)^{2}+\left(\sigma_{2}-\sigma_{3}\right)^{2}+\left(\sigma_{1}-\sigma_{3}\right)^{2}\right) / 2}
$$

where $\sigma_{1}, \sigma_{2}$ and $\sigma_{3}$ are main stresses $\left(\sigma_{1} \geq \sigma_{2} \geq \sigma_{3}\right)$.

Thus, having data available from $S$ sensors in the $j$-th time interval $\Delta T_{j}$, it is possible to restore both the values at each time step of the external forces $R$ (Equation 1) and the equivalent stresses $\sigma_{e j}(t)$ arising in the investigated positions of interest (Equations 3 and 4). The values of the sensitivity coefficients $a_{s, r}$ and the components of the stress tensors $\overline{\boldsymbol{\sigma}}_{*}^{(r)}$ required for this calculation can be obtained by the finite element method.

\subsection{Results of applying the method}

During the numerical experiments the problem of calculating the accumulated damage was realised for the first component generation (Figure 3, a). The time variation of the stress-strain state of the given component is due to the action of three mutually perpendicular forces $F_{r}(t) ; r=\overline{1, R} ; R=3$, which are attached to the lower part of the component as depicted. The part is rigidly fixed on the supporting cylindrical surface $O$.

The physical and geometric characteristics of the material of the component are known: $\sigma_{U}=274 \mathrm{MPa}$; $\sigma_{-1}=55 \mathrm{MPa} ; \sigma_{Y}=112 \mathrm{MPa}$. The forces $F_{r}(t)$ can be identified by the data from four sensors $(S=4)$ measuring the deformations $E_{S}(t), s=\overline{1, S}$ in the zones indicated in Figure 3, a, labelled by black rectangles A, B, C and D. The choice of these zones, the measuring axes of the sensors and the possible positions of failure with a maximum fatigue damage (point I in the Figure 3, a) were determined by the finite element method (Figure 1, b, for the case $F_{2}=1$ ) based on the analysis of the results of $R$ calculations of the distributions of the main directions of deformations in the component by the application of the $r$-th unit force $\left(F_{r}=1, F_{j \neq r}=0, r=\overline{1, R}\right)$.

The dimensions and the order of the finite elements were chosen from the condition of convergence of the results. The values of the elements of the matrix of coefficients $\mathbf{A}$ and the vector $\overline{\boldsymbol{\sigma}}^{(r)}$ were determined by averaging the strain values and mechanical stresses in the nodes of the calculated regions (A, B, C, D and I) by the finite element model. The following results were obtained (Lachmayer et al., 2018):

$$
\mathbf{A}=10^{-8}\left[\begin{array}{ccc}
-4.4 & 29.8 & -3.4 \\
1.24 & 17.6 & -1.0 \\
9.63 & 1.24 & -3.9 \\
0.76 & -7.1 & 6.43
\end{array}\right] ; \quad \begin{gathered}
\overline{\boldsymbol{\sigma}}^{(1)} \approx\left\{\begin{array}{lllllll}
1027 & 660 & 2895 & -38 & 65 & -1509
\end{array}\right\} \mathrm{Pa} \\
\overline{\boldsymbol{\sigma}}^{(3)} \approx\left\{\begin{array}{lllllll}
-443 & -218 & -1176 & -31 & -59 & 635
\end{array}\right\} \mathrm{Pa}
\end{gathered}
$$

The functions $E_{S}(t), s=\overline{1, S}$ used to identify the loads $F_{r}(t), r=\overline{1, R}$ acting on the component and the subsequent stress calculation were chosen as shown in Figure 3, c, curves 1-4, according to the obtained 
experimentally data. The duration of the load unit under investigation $\Delta T$ is $200 \mathrm{~s}$, the time step $\Delta t$ is constant and equals to $0.1 \mathrm{~s}\left(t_{m}=m \Delta t, m=0,1, \ldots, \Delta T / \Delta t\right)$.
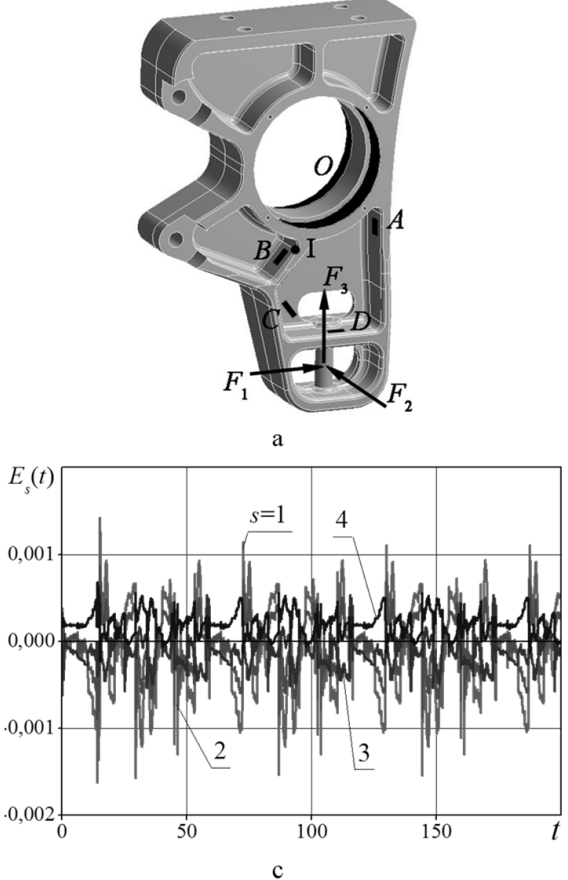

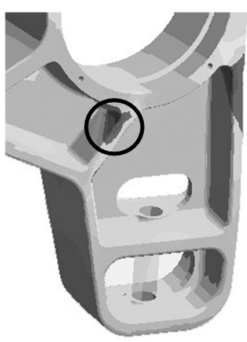

$F_{2}=1 ; F_{2}=1$

$\mathrm{b}$

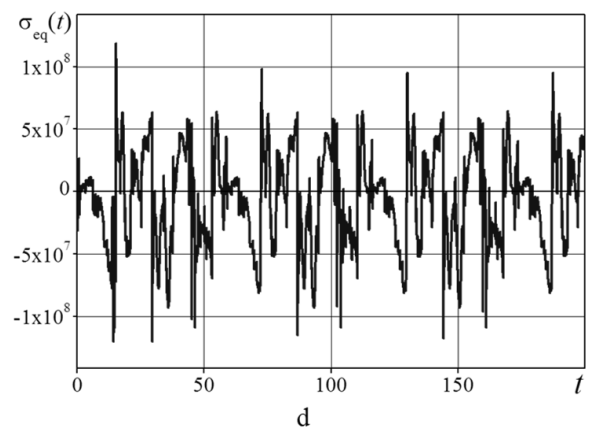

Figure 3. Example of using the method: a) first generation component with defined sensors positions; b) positions of a maximum fatigue damage;

c) experimentally obtained values of the deformation of the component at given positions; d) history of stress changes

Having the values $\left\{\mathbf{E}_{m}\right\}_{s}=E_{S}\left(t_{m}\right)$ (Figure 3, c), the matrix $\mathbf{A}$ and the vectors $\overline{\boldsymbol{\sigma}}^{(r)}, r=\overline{1, R}$, the approximate values of the function $\sigma_{e}\left(t_{m}\right)$ were determined at the calculated moments of time $t=t_{m}$. The results of the calculation are depicted in Figure 3, d. The subsequent processing according to the equations of the preceding paragraph gave a resulting fatigue damage of $\Delta D \approx 6.6 \cdot 10^{-4}$.

\section{Conclusion}

The approach of storage and transfer of information attained during the life cycle of a component with the goal of a subsequent use to develop a new generation of a product is called Technical Inheritance. The important aspect of this developed approach is the organization and support of an algorithmic data feedback of life cycle information. Thus, the design of a new generation of a product is based on information purposefully collected during the manufacturing and usage of previous generations.

The advantage and one of the main objectives of TI is the creation of the next generation of a component improvingly adapted to the requirements of the environment and based on collected usage data of previous generations of components.

The developed approach includes a method to analyse the current state of a component and to predict its residual life. The advantages of the method presented are the possibility of estimating the fatigue damage accumulated by the component according to the data from the applied sensors as well as through the described algorithm, the possibility to approximately restore the history of the stress state change at pre-selected points and to collect data about the acting component loads and ranges of their variations. These values can be used to improve the calculation of the residual life by computational methods and for the development of new generations of components. 


\section{References}

Barton, S., Mroz, G., Reimche, W. and Maier, H.J. (2016), "Inherent Load Measurement and Component Identification by multi-dimensional Coded Data in the Component's Subsurface Region", Procedia Technology, Vol. 26, pp. 537-543. https://doi.org/10.1016/j.protcy.2016.08.067

Bian, L., Gebraeel, N. and Kharoufeh, J.P. (2015), "Degradation modeling for real-time estimation of residual lifetimes in dynamic environments", IIE Transactions, Special Issue: Quality and Reliability Engineering, Vol. 47 No. 5, pp. 471-486. https://doi.org/10.1080/0740817X.2014.955153

Collins, J.A. (1993), Failure of materials in mechanical design: Analysis, prediction, prevention, John Wiley \& Sons, New York.

Demminger, C., Mozgova, I., Quirico, M., Uhlich, F., Denkena, B. et al. (2016), "The Concept of Technical Inheritance in Operation: Analysis of the Information Flow in the Life Cycle of Smart Products", Procedia Technology, Vol. 26, pp. 79-88. https://doi.org/10.1016/j.protcy.2016.08.012

Denkena, B., Lachmayer, R., Ostermann, J. and Nyhuis, P. (2016), "Datenaustausch in den verschiedenen Phasen des Lebenszyklus von smarten Produkten”, VDI IT\&Production, Vol. 1-2.

Gembarski, P.C., Sauthoff, B., Brockmöller, T. and Lachmayer, R. (2016), "Operationalization of Manufacturing Restrictions for CAD and KBE-Systems”, Proceedings of the DESIGN 2016 / 14th International Design Conference, Dubrovnik, Croatia, May 16-19, 2016, pp. 621-630.

Goto, S. and Tsukamoto, K. (2012), "On-line residual life prediction including outlier elimination for condition based maintenance", International Journal of Innovative Computing, Information and Control, Vol. 8 No. 3(B), pp. 2193-2202.

Gottwald, P. (2016), Prozess einer generationsübergreifenden Produktentwicklung durch technische Vererbung, $\mathrm{PhD}$ thesis, Leibniz Universität Hannover, Berichte aus dem IPeG, PZH-Verlag, Garbsen.

Köhler, M., Jenne, S., Pötter, K. and Zenner, H. (2012), Zählverfahren und Lastannahme in der Betriebsfestigkeit, Springer-Verlag Berlin-Heidelberg.

Lachmayer, R., Mozgova, I. and Gottwald, P. (2015), "Formulations of Paradigms of Technical Inheritance", Proceedings of the 20th International Conference on Engineering Design (ICED15), Volume 8: Innovation and Creativity, Milan, Italy, July 27-30, 2015, pp. 271-278.

Lachmayer, R., Mozgova, I., Reimche, W., Colditz, F., Mroz, G. and Gottwald, P. (2014a), “Technical Inheritance: A Concept to Adapt the Evolution of Nature to Product Engineering”, Procedia Technology, Vol. 15, pp. 178187. https://doi.org/10.1016/j.protcy.2014.09.070

Lachmayer, R., Mozgova, I., Sauthoff, B. and Gottwald, P. (2013), "Product Evolution and Optimization based on Gentelligent Components and Product Life Cycle Data", Proceedings of the 23rd CIRP Design Conference, Bochum, Germany, March 11-13, 2013, Springer Verlag, Berlin-Heidelberg, pp. 687-694. https://doi.org/10.1007/978-3-642-30817-8_67

Lachmayer, R., Mozgova, I., Sauthoff, B. and Gottwald, P. (2014b), "Evolutionary Approach for an Optimized Analysis of Product Life Cycle Data", Proceedings of the 2nd International Conference on System-Integrated Intelligence: Challenges for Product and Production Engineering (SysInt 2014), Bremen, Germany, July 2-4, 2014, pp. 359-368. https://doi.org/10.1016/j.protcy.2014.09.090

Lachmayer, R., Yanchevskyi, I., Gottwald, P. and Mozgova, I. (2018), "Identification of several non-stationary loads applied to an elastically deformed element of constructions", Journal of Applied and Computational Mechanics (in Review).

Mozgova, I., Barton, S., Demminger, C., Miebach, T., Taptimthong, P. et al. (2017), “Technical inheritance: Information basis for the identification and development of product generations", Proceedings of the 21st International Conference on Engineering Design (ICED 17), Vol. 6: Design Information and Knowledge, Vancouver, Canada, August 21-25, 2017, pp. 91-100.

Scheidel, W., Mozgova, I. and Lachmayer, R. (2017), "Product Data Management in the Context of Industry 4.0", Proceedings of the 59th Ilmenau Scientific Colloquium, Vol. 59 Engineering for a Changing World (ilmedia), Ilmenau, Germany, September, 11-15, 2017.

Schubert, R., Mozgova, I. and Lachmayer, R. (2013), "Moderne Methoden der datenbasierten Prognose und deren praktische Anwendung bei Aussagen zur technischen Zuverlässigkeit: Schnelligkeit und Genauigkeit von Zuverlässigkeitsprognosen", VDI-Berichte, Vol. 2210, pp. 159-176.

Zhou, R.R., Serban, N. and Gebraeel, N. (2011), "Degradation modeling applied to residual lifetime prediction using functional data analysis", The Annals of Applied Statistics, Vol. 5 No. 2B, pp. 1586-1610. https://doi.org/10.1214/10-AOAS448

Dr. Iryna Mozgova

Leibniz Universität Hannover, Institute of Product Development (Institut für Produktentwicklung und Gerätebau) Welfengarten 1A, 30167 Hannover, Germany

Email:mozgova@ipeg.uni-hannover.de 